\title{
Disease-modifying agents in progressive multiple sclerosis: Management of 100 patients at Louis Stokes Cleveland VAMC, Spinal Cord Injury Division
}

\author{
Saisho Mangla, BS; ${ }^{1}$ Seema Jain; ${ }^{1}$ Stephen Selkirk, $\mathrm{MD}, \mathbf{P h D}^{2-3 *}$ \\ Departments of ${ }^{1}$ Neuroscience and ${ }^{2}$ Neurology, Case Western Reserve School of Medicine, Cleveland, OH; ${ }^{3}$ Louis \\ Stokes Cleveland Department of Veterans Affairs Medical Center, Spinal Cord Injury Division, Cleveland, $\mathrm{OH}$
}

\begin{abstract}
Multiple sclerosis (MS) is a chronic disease in which disability progresses over time. Progressive forms of MS have a poor prognosis, are associated with greater levels of disability and, unfortunately, are unresponsive to current treatments. Here, we have reviewed the management of 100 patients with MS. The majority of these patients had progressive disease, Expanded Disability Status Scale scores $>6$, and extensive medical complications. A significant number of patients in this cohort were also treated with MS disease-modifying agents that lack efficacy in patients with progressive disease. Although these drugs are relatively safe, their use here is significantly costly to the healthcare system, with limited benefit to patients. We suggest that these drugs be discontinued in these patients and resources be directed toward symptomatic treatment, rehabilitation needs, and management of medical complications until drugs with proven efficacy become available.
\end{abstract}

Key words: central nervous system, Copaxone, disease-modifying agents, EDSS, interferon beta, primary progressive multiple sclerosis, rehabilitation, relapsing-remitting multiple sclerosis, secondary progressive multiple sclerosis, spinal cord injury.

\section{INTRODUCTION}

Multiple sclerosis (MS) is a chronic and progressive disease of the central nervous system (CNS), in which physical disability progresses over time. It remains the most common cause of nontraumatic neurological disability in young adults and is a significant cause of dis- ability in the U.S. veteran population. The indirect and direct medical costs are an estimated $\$ 12$ billion annually and include provider healthcare, physical therapy, rehabilitation needs, and medications but also lost productivity, missed workdays, and loss of individual earnings. Healthcare costs, as expected, increase with increasing disability, reflecting frequent complications that occur in disabled patients regardless of the underlying disease [1].

Four distinct clinical courses of MS have been defined [2], but the majority of the disease commences with relapsing-remitting MS (RRMS). The relapsingremitting course is presumably driven by recurrent waves of focal inflammation into the brain, manifesting as acute disability, which improves, to a varying degree, after the

\footnotetext{
Abbreviations: $\mathrm{CNS}=$ central nervous system, DMA = disease-modifying agent, EDSS = Expanded Disability Status Scale, FDA = Food and Drug Administration, LSCVAMC = Louis Stokes Cleveland Department of Veterans Affairs Medical Center, MRI = magnetic resonance imaging, $\mathrm{MS}=$ multiple sclerosis, PPMS = primary progressive MS, RRMS = relapsing-remitting MS, SCI = spinal cord injury, SPMS = secondary progressive MS, VA = Department of Veterans Affairs.

* Address all correspondence to Stephen Selkirk, MD, PhD; Louis Stokes Cleveland VA Medical Center, Spinal Cord Injury Division, 10701 East Blvd, Mayfield Heights, OH 44124; 216-791-3800; fax: 216-707-5902.

Email: stephenselkirkmdphd@gmail.com

DOI:10.1682/JRRD.2010.10.0200
} 
exacerbation. These episodes of acute focal CNS inflammation are mirrored in the magnetic resonance imaging (MRI) of patients with RRMS as T2 hyperintensities, although some of these episodes and MRI sequelae may be clinically silent. Diagnostic criteria are based on the separation of lesions in time and space (different anatomical locations within the CNS). This criterion has not changed, although multiple revisions to the original criteria [3] have included the use of MRI to support this basic clinical tenet [4]. In over half of these patients, the relapsing-remitting disease course will convert to secondary progressive MS (SPMS). Twenty-five years after the diagnosis of RRMS, 75 percent of patients will have converted to SPMS [5]. This change is identified clinically by the absence or nearabsence of relapses, with disability continuing to progress.

In contrast, approximately 7 to 20 percent of patients [6-7] have primary progressive MS (PPMS) characterized by neurological disability that progressively worsens over time in the absence of acute relapses. Over 80 percent of patients with PPMS will present with a slowly progressive spastic paraparesis. This subtype presumably lacks the intense punctate inflammatory events that result in exacerbations. Rather, PPMS is thought to be either neurodegenerative in nature or related to low-level persistent inflammation. The MRI findings in patients with PPMS are distinct because volume loss is more prominent with a lesser amount of T2 abnormalities compared with patients with RRMS. Diagnostic criteria have been described and include the exclusion of other causes, progression in the absence of relapses, a clinical syndrome consistent with the diagnosis, and supporting MRI and cerebral spinal fluid studies [8]. Interestingly, the clinical course of PPMS is nearly indistinguishable from SPMS. Over time, patients with these two subtypes are equally disabled. This result is true regardless of the severity of the initial relapsing-remitting course for patients with SPMS, suggesting that focal inflammatory events are perhaps less important in determining long-term disability levels [5,9-10]. Figure 1 gives the main characteristics of RRMS, SPMS, and PPMS.

Originally, the utility of defining these MS subtypes helped in recruiting appropriate patients for clinical trials. Currently, these distinctions are important because of differences in prognosis between subtypes, as well as differences in responsiveness to Food and Drug Administration (FDA)-approved MS medications. Medications target the inflammatory nature of the disease, and although they have demonstrated the capability to decrease the number of

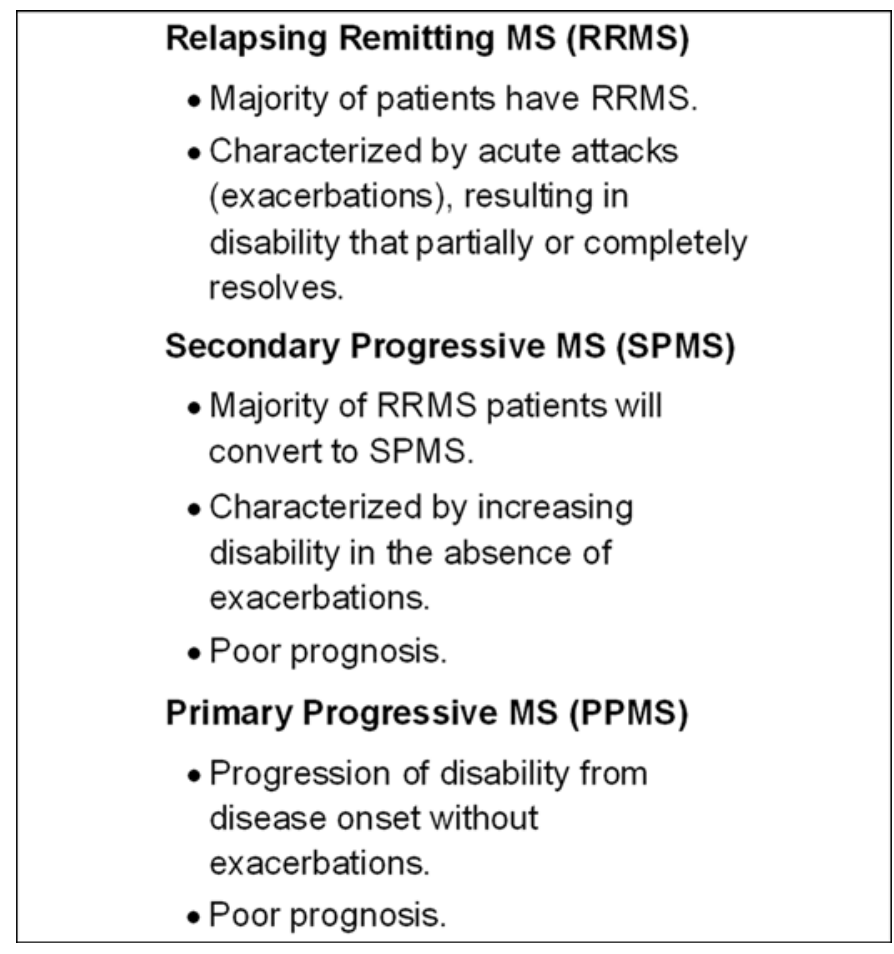

Figure 1.

RRMS, SPMS, and PPMS disease courses of multiple sclerosis (MS) and their main characteristics.

relapses a patient experiences, the evidence that these treatments significantly alter the disease course in terms of disability progression over time is weak [11]. Furthermore, little evidence exists to suggest that these medications are effective for patients with a progressive disease course.

Here, we present data regarding the management of 100 patients with MS cared for by a multidisciplinary team in the Spinal Cord Injury (SCI) Division of the Louis Stokes Cleveland Department of Veterans Affairs (VA) Medical Center (LSCVAMC), with a focus on the use of disease-modifying agents (DMAs).

\section{METHODS}

Institutional review board approval allowed us to identify patients with the diagnosis of MS from the SCI Division patient registry at the LSCVAMC. We extracted the diagnosis subtype from neurology clinic notes or through direct evaluation. By reviewing the medications that the LSCVAMC prescribed to the patient, we identified the current treatment. We determined the level of disability in 
patients using the Expanded Disability Status Scale (EDSS). The EDSS is the most widely used scale for determining disability and disease progression in patients with MS [12]. Patient progression in the EDSS score indicates disease progression and is an important measure in MS clinical trials. In this study, we inferred the EDSS score either from physical therapy evaluations that are completed annually by the SCI Division or through direct evaluation. We determined spasticity management by the presence of spasticity medications (Valium, baclofen, intrathecal baclofen, tizanidine, dantrolene) in the patients' medication list. Finally, we extracted demographic data from the electronic chart. We de-identified all data according to LSCVAMC policies.

\section{RESULTS}

\section{Characteristics of Spinal Cord Injury MS Cohort}

The SCI Division MS cohort is composed of patients with spinal cord involvement defined by having either neurogenic bowel or bladder and/or gait dysfunction attributed to a lesion in the spinal cord. Patients are usually referred to the SCI Division for rehabilitation needs (wheelchair, durable medical equipment, etc.) or continued management of neurogenic bowel or bladder. The majority of patients in our cohort were male (overall male-female ratio was 7:1). This ratio is significantly different from cohorts previously reported on and likely represents the male predominance of the veteran population. This male predominance decreases in the RRMS subgroup (male-female, 3:1) and increases in the PPMS (male-female, 33:1). The average age of the cohort was 58 years old, while the average age of patients with PPMS was 60 years old, SPMS 57 years old, and RRMS 53 years old (Table 1).

\section{Disability Profile}

Patients managed by the SCI Division had an average EDSS score of 6.6. The average EDSS score of patients with PPMS was higher (7.4), while the score of patients with RRMS was lower (4.5). The average EDSS score of patients with SPMS was 6.6. This generally reflects that spinal cord involvement, a prerequisite for the SCI Division, results in impaired gait and higher EDSS score (Table 1). Bladder dysfunction was found in $>90$ percent of patients and 100 percent of patients with PPMS, while bowel dysfunction was less common overall but was documented in nearly all patients with PPMS. Because of the level of disability, most patients (70\%) required spasticity
Table 1.

Cohort characteristics are classified according to multiple sclerosis (MS) subtype.

\begin{tabular}{|c|c|c|c|c|}
\hline Characteristic & PPMS & SPMS & RRMS & All \\
\hline Sex (F:M) & 1:33 & $1: 6$ & $1: 3$ & $1: 7$ \\
\hline EDSS (score) & 7.4 & 6.6 & 4.5 & 6.6 \\
\hline Age (yr) & 60 & 57 & 53 & 58 \\
\hline \multicolumn{5}{|l|}{ Race (\%) } \\
\hline Caucasian & 60 & 64 & 53 & 63 \\
\hline Black & 16 & 6 & 20 & 12 \\
\hline Unknown & 21 & 25 & 26 & 22 \\
\hline $\begin{array}{l}\text { EDSS = Expanded } \mathrm{I} \\
\text { mary progressive } \mathrm{M} \\
\text { progressive MS. }\end{array}$ & $\begin{array}{l}\text { ity St } \\
\mathrm{MS}\end{array}$ & $\begin{array}{l}\text { e, F = } \\
\text { g-ren }\end{array}$ & $\begin{array}{l}\mathrm{M}=\mathrm{m} \\
\mathrm{AS}, \mathrm{SPI}\end{array}$ & $\begin{array}{l}\mathrm{MS}= \\
\text { econd }\end{array}$ \\
\hline
\end{tabular}

management (data not shown). Many of these patients were prescribed more than one medication for spasms, and 6 percent had an intrathecal baclofen pump for spasticity management.

To identify the complications associated with disease and disability, we determined the reason for the last hospital admission. The most common presentation leading to acute hospital admission was grouped into general medicine issues, including chest pain, wound management, shortness of breath, and lower-limb edema. The second most common reason for acute hospital admission was complications of neurogenic bladder, including urinary tract infection, urinary retention, traumatic hypospadias, stent placement for renal calculi, and cystolitholapaxy. We documented acute admissions directly associated with MS disease activity in 13 admissions, 8 of these were in patients with RRMS (Table 2).

\section{MS Subtypes}

The majority of patients managed by the SCI Division had a diagnosis of SPMS or PPMS (85\%). Only 15 percent of patients had RRMS and qualified for care from the SCI Division. This finding is consistent with the concept that patients with progressive disease have higher disability levels and more spinal cord involvement than patients with RRMS. However, the percentage of patients with PPMS was notable compared with that of patients with SPMS (Figure 2). Clearly, if RRMS represents the majority of patients diagnosed, then the progressive disease subtypes should be overrepresented by SPMS. In our cohort, the ratio of SPMS to PPMS was 1.3:1.0. Whether this finding resulted from the skewing of sex in the VA population to male predominance or from a higher percentage of PPMS among veterans is unclear. 
Table 2.

Diagnosis associated with patient's last hospital admission was recorded. Majority of admissions were associated with medical issues or complications of neurogenic bladder. Minority of admissions were related directly to multiple sclerosis (MS) disease activity and concentrated in relapsing-remitting MS subgroup.

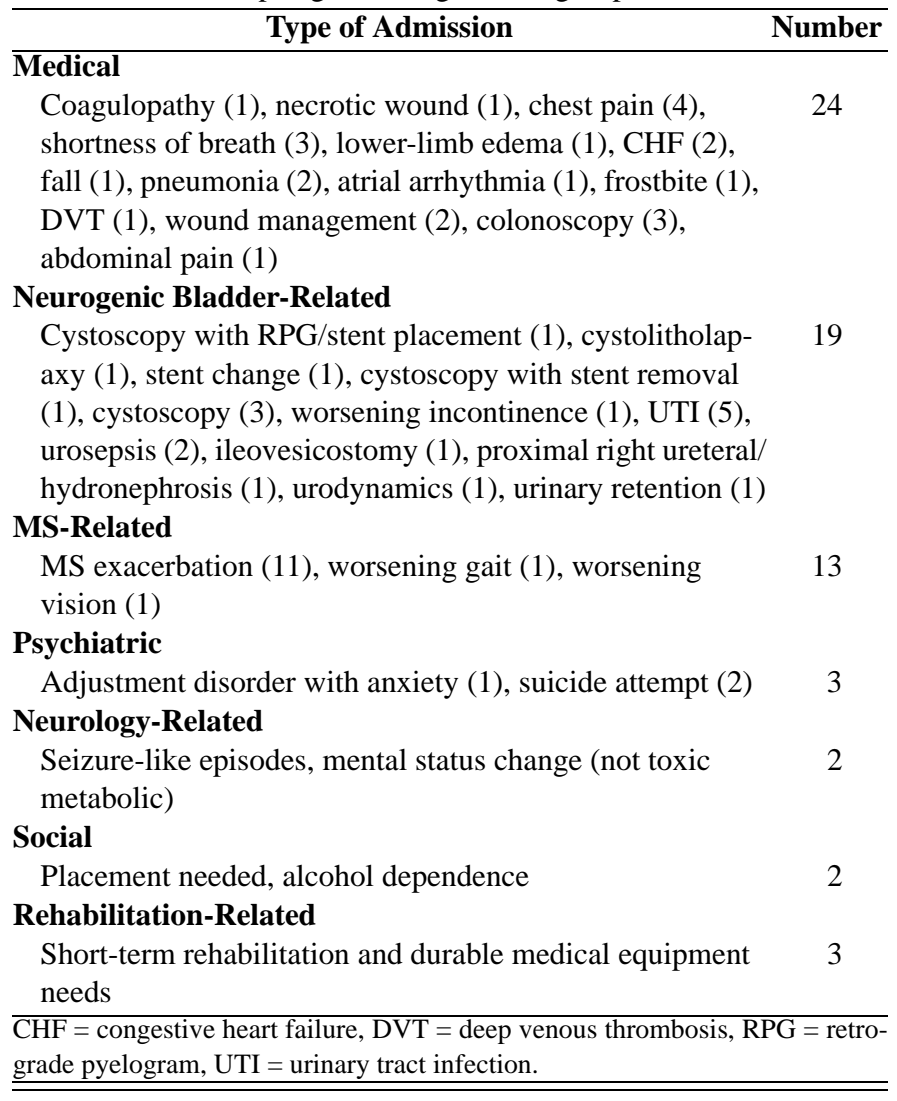

\section{Management of MS with FDA-Approved Disease- Modifying Agents}

All patients taken together, the most common treatment administered was Copaxone (21\%), followed by interferon $\beta$-1a (10\%), methotrexate (8\%), Copaxone with methotrexate (8\%), and interferon $\beta$-1b (5\%). Notably, 37 percent of patients received no treatment at all. Nearly all patients with RRMS were treated with one of the FDA-approved DMAs (Copaxone, Rebif, interferon $\beta$-1a, or interferon $\beta$-1b) (Figure 2). One patient with RRMS had received Tysabri.

In contrast, no patients with SPMS were currently being treated with Novantrone, which is FDA-approved for this subclass. Only two patients followed by the SCI Division had ever received this drug. The majority of patients with SPMS were treated with Copaxone (18\%), interferon $\beta$-1a (12\%), methotrexate (8\%), Copaxone plus methotrexate (10\%), or interferon $\beta$-1a plus methotrexate (a)

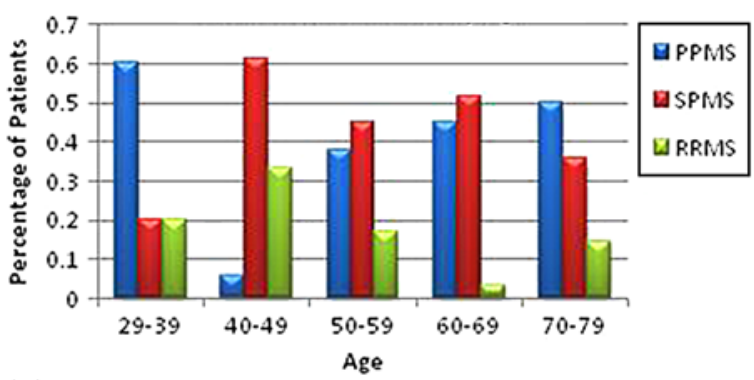

(b)

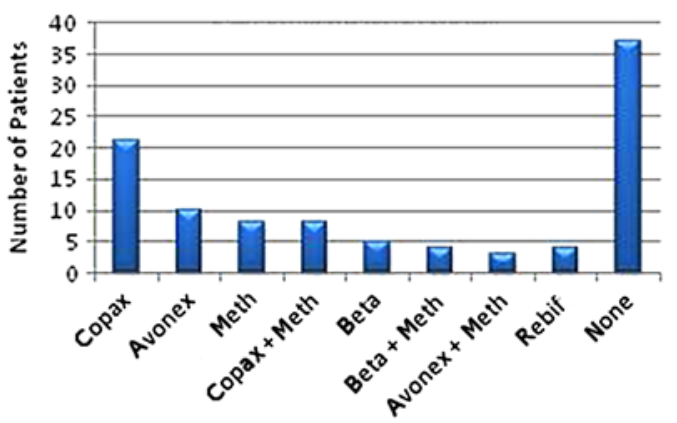

(c)

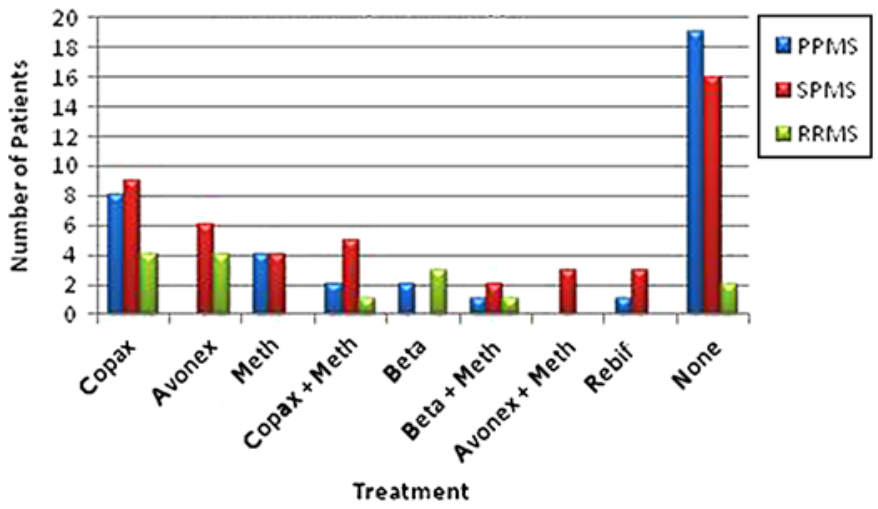

Figure 2.

Spinal Cord Injury Division multiple sclerosis (MS) cohort was mainly represented by (a) patients with progressive disease because only $15 \%$ were categorized as relapsing-remitting MS (RRMS). Number of patients prescribed various disease-modifying agents (DMAs) was determined for (b) all patients and (c) each MS subtype. All patients with RRMS were prescribed DMA, while Copaxone (Copax) was most commonly prescribed DMA for patients with progressive disease, although majority of patients were not treated. Beta $=$ Betaseron, Meth $=$ methotrexate, PPMS $=$ primary progressive MS, SPMS = secondary progressive MS.

(6\%). The majority of patients with PPMS were not treated (51\%). However, 21 percent of patients were prescribed Copaxone and another 8 percent received Copaxone plus methotrexate (Figure 2). 


\section{DISCUSSION}

Progressive forms of MS are difficult to manage. Multiple reports describing the natural history of PPMS suggest a worse prognosis and more significant disability levels compared with patients with RRMS. SPMS is known to follow a similar course, with advancing levels of disability $[7,13]$. The progressive disease course is defined by the absence or near absence of clinically identifiable relapses, making this diagnosis intuitively less responsive to FDA-approved medications that decrease relapse rates in patients with MS. With these considerations in mind, we reviewed a cohort of patients whom the LSCVAMC SCI Division manages and most have progressive disease. Although cohorts of veterans and nonveterans with MS have been well described, progressive disease has not been extensively defined in veterans, particularly in terms of the rehabilitation needs, medical comorbidities, and use of DMAs.

Our cohort consisted primarily of male subjects because of the male predominance in military service. Therefore, comparing the demographics of our cohort with those that have been described was difficult. Having said this, some details can be extrapolated and compared with other cohorts. Specifically, the subgroup of patients with PPMS was clearly more disabled than the subgroup with RRMS or SPMS. This finding agrees with the concept that the diagnosis of PPMS confers a worse prognosis than RRMS. Also, as we expected, the RRMS subgroup had relatively more female patients than the PPMS subgroup, which is thought to affect male and females equally. Finally, the vast majority of patients were Caucasian, which is also consistent with cohorts described previously [14-15].

The patients in the cohort were generally referred to the SCI Division because of extensive rehabilitation needs and medical complications. Studies have demonstrated that comprehensive multidisciplinary centers offer care that improves patients' satisfaction and quality of life [16]. However, these centers are often urban-based and not realistically routinely accessible to most patients. More commonly, patients receive care from a variety of different disciplines, including internists, physiatrists, and neurologists. Our study confirms the concept that patients with progressive disease have a significant degree of disability and related medical comorbidities [13]. Medical admissions and admissions for complications from neurogenic bladder were three times more common than admissions for disease-specific events, such as exacerbation or worsening neurological function, further emphasizing the need for multidisciplinary management of patients with progressive MS.

The level of disability in our cohort resulted in extensive rehabilitation and medical services. However, the management of the disease process itself in patients not only with progressive disease but also with advanced disease was notable. Specifically, more than one in four patients with progressive disease was prescribed Copaxone. Two large-scale trials have been completed to study its effectiveness in progressive disease. The first was a trial that included 109 patients with either SPMS or PPMS. It failed to demonstrate efficacy for the drug in slowing the rate of disease progression [17]. Secondary analysis suggested the possibility that patients with PPMS may have responded to treatment, and subsequently, a large-scale, multicenter trial, PROMiSe [18], enrolling only patients with PPMS, was undertaken. This trial enrolled 943 patients with PPMS at multiple centers worldwide but failed to demonstrate efficacy in preventing disability progression over time. The study was halted prematurely because of perceived futility [18]. This failure was surprising to many, given the alleged claim that Copaxone modifies chronic inflammation and decreases MRI indicators of neurodegenration [19].

We propose several reasons why so many patients are still being treated with Copaxone, even though welldesigned trials have demonstrated a lack of efficacy. First, physicians may not be familiar with the complex data and the FDA indications of this drug; second, progressive disease may instill a sense of hopelessness for both patient and provider. The FDA approval and favorable side-effect profile make Copaxone an easy choice. Third, often, as patients convert to SPMS from RRMS, providers view this decline as a therapeutic failure of interferon $\beta$ and therefore convert patients to Copaxone. As patients continue to decline and progress with SPMS, they are continued on their last prescribed DMA, even though it is not relevant.

Similar to Copaxone use, almost one in four patients with progressive disease was prescribed one of the three available interferon $\beta$ s. No evidence supports the use of this drug in PPMS, and data regarding its use in SPMS is suspect. Four large-scale studies have been conducted to determine the efficacy of interferon $\beta$ in patients with progressive disease. The first, the European SPMS trial, was the only of these to demonstrate efficacy, showing a 
significant delay in disability progression by 9 months in treated patients [20]. However, three subsequent trials have failed to recapitulate this success, making the clinical decision regarding the use of this drug in progressive disease complex [21-23]. Extensive post hoc analysis suggests that unless a patient is having active relapses in the SPMS state, no evidence exists to support efficacy.

The inappropriate use of Copaxone and interferon $\beta$ in progressive MS not only exposes patients to ineffective therapies with potentially harmful side effects but also represents an unnecessary economic cost to patients and the healthcare system. In the VA system, the average cost for a 1-year treatment of Copaxone is $\$ 18,052.84$ for each patient. In our cohort alone, this amount represents $>\$ 523,508$ a year spent on therapy that is ineffective. The average cost of Avonex, Betaseron, and Rebif is $\$ 13,731$, $\$ 10,038$, and $\$ 14,073$ a year, respectively, for each patient. In our cohort, this cost represents an additional $\$ 325,137$ a year. In total, $>\$ 800,000$ a year was spent on therapy that lacks proof of efficacy in well-designed, multicenter, placebo-controlled, blinded studies. In contrast, medications like methotrexate that have been used with similar efficacy cost $\$ 8.36$ a year. These costs vary from year to year based on negotiated contracts but have remained relatively stable over time. Studies of costs for these medications in the private sector have shown an increase of 13.6 percent annually from 2004 to 2007, with stable use [24]. This finding suggests that the costs of DMAs are not likely to decrease in the near future.

This study has multiple limitations, including that it is a retrospective chart review study and is limited to a cohort of 100 patients in the Cleveland VA system. The cost of DMAs is based on nationwide contracted information and can be generalized to the entire VA system, but the use of DMAs at other facilities was not reviewed here. Furthermore, private sector use was not reviewed and our conclusions cannot necessarily be generalized to these patients either. Finally, our conclusions pertain only to patients with progressive MS and should not limit the use of DMAs in patients with RRMS. The exact time of conversion of RRMS to SPMS can be difficult to identify, and therefore, if the exact subtype of MS remains in question, patients should continue taking DMAs until their progressive course becomes clinically apparent.

\section{CONCLUSIONS}

In summary, the LSCVAMC SCI Division cohort consisting mostly of patients with progressive disease, which was disproportionately male, had complex medical issues requiring interdisciplinary care and multiple hospital admission for non-MS-related medical reasons. Many patients, although significantly disabled, were continued on MS medications, which have been shown to be ineffective in altering the disease course of PPMS and SPMS. This finding underscores the importance of multidisciplinary management of patients with progressive MS, as well as a need to resist treating progressive subtypes of MS with medications that lack efficacy. Rather, the focus should be on symptomatic treatment and preventing complications in patients who are chronically disabled until medications with proven efficacy become available.

\section{ACKNOWLEDGMENTS}

\section{Author contributions:}

Acquisition of data: S. Jain.

Analysis and interpretation of data: S. Mangla, S. Jain.

Drafting of Manuscript: S. Selkirk.

Critical revision of manuscript for important intellectual content: S. Selkirk.

Statistical Analysis: S. Mangla, S. Jain.

Financial Disclosure: The authors have declared that no competing interests exist.

Funding/Support: This material was unfunded at the time of manuscript preparation.

Institutional Review: The authors received institutional review board approval from the LSCVAMC.

Participant Follow-Up: The authors do not plan to inform the participants of the publication of this study.

Disclaimer: The views expressed in this article are those of the authors and do not necessarily represent the position or policy of the VA or the Department of Defense.

\section{REFERENCES}

1. Richards RG, Sampson FC, Beard SM, Tappenden P. A review of the natural history and epidemiology of multiple sclerosis: Implications for resource allocation and health economic models. Health Technol Assess. 2002;6(10):1-73. [PMID: 12022938]

2. Lublin FD, Reingold SC. Defining the clinical course of multiple sclerosis: Results of an international survey. National Multiple Sclerosis Society (USA) Advisory Committee on 
Clinical Trials of New Agents in Multiple Sclerosis. Neurology. 1996;46(4):907-11. [PMID: 8780061]

3. Schumacher GA, Beebe G, Kibler RF, Kurland LT, Kurtzke JF, McDowell F, Nagler B, Sibley WA, Tourtellotte WW, Willmon TL. Problems of experimental trials of therapy in multiple sclerosis: Report by the panel on the evaluation of experimental trials of therapy in multiple sclerosis. Ann N Y Acad Sci. 1965;122:552-68. [PMID: 14313512] http://dx.doi.org/10.1111/j.1749-6632.1965.tb20235.x

4. Polman CH, Reingold SC, Edan G, Filippi M, Hartung HP, Kappos L, Lublin FD, Metz LM, McFarland HF, O’Connor PW, Sandberg-Wollheim M, Thompson AJ, Weinshenker BG, Wolinsky JS. Diagnostic criteria for multiple sclerosis: 2005 revisions to the "McDonald Criteria." Ann Neurol. 2005;58(6):840-46. [PMID: 16283615] http://dx.doi.org/10.1002/ana.20703

5. Runmarker B, Anderson O. Prognostic factors in a multiple sclerosis incidence cohort with twenty-five years followup. Brain. 1993;116(Pt 1):117-34. [PMID: 8453453] http://dx.doi.org/10.1093/brain/116.1.117

6. Anderson PB, Waubant E, Gee L, Goodkin DE. Multiple sclerosis that is progressive from the time of onset: Clinical characteristics and progression of disability. Arch Neurol. 1999;56(9):1138-42. [PMID: 10488816] http://dx.doi.org/10.1001/archneur.56.9.1138

7. Cottrell DA, Kremenchutzky M, Rice GP, Koopman WJ, Hader W, Baskerville J, Ebers GC. The natural history of multiple sclerosis: A geographically based study: 5. The clinical features and natural history of primary progressive multiple sclerosis. Brain. 1999;122(Pt 4):625-39. [PMID: 10219776$]$ http://dx.doi.org/10.1093/brain/122.4.625

8. Thompson AJ, Montalban X, Barkhof F, Brochet B, Filippi M, Miller DH, Polman CH, Stevenson VL, McDonald WI. Diagnostic criteria for primary progressive multiple sclerosis: A position paper. Ann Neurol. 2000;47(6):831-35. [PMID: 10852554] http://dx.doi.org/10.1002/15318249(200006)47:6<831::AID-ANA21>3.0.CO;2-H

9. Kantarci O, Siva A, Eraksoy M, Karabudak R, Sütla N, Aaolu J, Turan F, Ozmenolu M, Torul E, Demirkiran M. Survival and predictors of disability in Turkish MS patients; Turkish Multiple Sclerosis Study Group (TUMSSG). Neurology. 1998;51(3):765-72. [PMID: 9748024]

10. Confavreux C, Vukusic S, Moreau T, Adeleine P. Relapses and progression of disability in multiple sclerosis. N Engl J Med. 2000;343(20):1430-38. [PMID: 11078767] http://dx.doi.org/10.1056/NEJM200011163432001

11. Kappos L, Freedman MS, Polman CH, Edan G, Hartung HP, Miller DH, Montalbán X, Barkhof F, Radü EW, Metzig C, Bauer L, Lanius V, Sandbrink R, Pohl C; BENEFIT Study Group. Long-term effect of early treatment with interferon beta-1b after a first clinical event suggestive of multiple sclerosis: 5-year active treatment extension of the phase 3 BENEFIT trial. Lancet Neurol. 2009;8(11):987-97.

[PMID: 19748319]

http://dx.doi.org/10.1016/S1474-4422(09)70237-6

12. Kurtzke JF. Rating neurologic impairment in multiple sclerosis: An Expanded Disability Status Scale (EDSS). Neurology. 1983;33(11):1444-52. [PMID: 6685237]

13. McDonnell GV, Hawkins SA. Clinical study of primary progressive multiple sclerosis in Northern Ireland, UK. J Neurol Neurosurg Psychiatry. 1998;64(4):451-54. [PMID: 9576534]

14. Sumelahti ML, Tienari PJ, Hakama M, Wikström J. Multiple sclerosis in Finland: Incidence trends and differences in relapsing remitting and primary progressive disease courses. J Neurol Neurosurg Psychiatry. 2003;74(1):25-28. [PMID: 12486261$]$ http://dx.doi.org/10.1136/jnnp.74.1.25

15. Tremlett H, Paty D, Devonshire V. The natural history of primary progressive MS in British Columbia, Canada. Neurology. 2005;65(12):1919-23. [PMID: 16380613] http://dx.doi.org/10.1212/01.wnl.0000188880.17038.1d

16. Wu N, Minden SL, Hoaglin DC, Hadden L, Frankel D. Quality of life in people with multiple sclerosis: Data from the Sonya Slifka Longitudinal Multiple Sclerosis Study. J Health Hum Serv Adm. 2007;30(3):233-67. [PMID: 18236703]

17. Bornstein MB, Miller A, Slagle S, Weitzman M, Drexler E, Keilson M, Spada V, Weiss W, Appel S, Rolak L, Harati Y, Brown S, Arnon R, Jacobsohn I, Teitelbaum D, Sela M. A placebo-controlled, double-blind, randomized, two-center, pilot trial of Cop 1 in chronic progressive multiple sclerosis. Neurology. 1991;41(4):533-39. [PMID: 2011253]

18. Wolinsky JS, Narayana PA, O’Connor P, Coyle PK, Ford C, Johnson K, Miller A, Pardo L, Kadosh S, Ladkani D; PROMiSe Trial Study Group. Glatiramer acetate in primary progressive multiple sclerosis: Results of a multinational, multicenter, double-blind, placebo-controlled trial. Ann Neurol. 2007; 61(1):14-24. [PMID: 17262850] http://dx.doi.org/10.1002/ana.21079

19. Filippi M, Rovaris M, Rocca MA, Sormani MP, Wolinsky JS, Comi G; European/Canadian Glatiramer Acetate Study Group. Glatiramer acetate reduces the proportion of new MS lesions evolving into “black holes.” Neurology. 2001; 57(4):731-33. [PMID: 11524494]

20. Placebo-controlled multicentre randomised trial of interferon beta-1b in treatment of secondary progressive multiple sclerosis. European Study Group on interferon beta-1b in secondary progressive MS. Lancet. 1998;352(9139): 1491-97. [PMID: 9820296]

21. Panitch H, Miller A, Paty D, Weinshenker B; The North American Study Group on Interferon beta-1b in Secondary Progressive MS. Interferon beta-1b in secondary progressive MS: Results from a 3-year controlled study. Neurology. 2004;63(10):1788-95. [PMID: 15557491] 
22. Cohen JA, Cutter GR, Fischer JS, Goodman AD, Heidenreich FR, Kooijmans MF, Sandrock AW, Rudick RA, Simon JH, Simonian NA, Tsao EC, Whitaker JN; IMPACT Investigators. Benefit of interferon beta-1a on MSFC progression in secondary progressive MS. Neurology. 2002;59(5):679-87.

[PMID: 12221157]

23. Secondary Progressive Efficacy Clinical Trial of Recombinant Interferon-beta-1a in MS (SPECTRIMS) Study Group. Randomized controlled trial of interferon-beta-1a in secondary progressive MS: Clinical results. Neurology. 2001; 56(11):1496-1504. [PMID: 11402106]

24. Kunze AM, Gunderson BW, Gleason PP, Heaton AH, Johnson SV. Utilization, cost trends, and member cost-share for self-injectable multiple sclerosis drugs-Pharmacy and medical benefit spending from 2004 through 2007. J Manag Care Pharm. 2007;13(9):799-806. [PMID: 18062731]
Submitted for publication October 14, 2010. Accepted in revised form May 11, 2011.

This article and any supplementary material should be cited as follows:

Mangla S, Jain S, Selkirk S. Disease-modifying agents in progressive multiple sclerosis: Management of 100 patients at Louis Stokes Cleveland VAMC, Spinal Cord Division. J Rehabil Res Dev. 2011;48(10):1223-30.

DOI:10.1682/JRRD.2010.10.0200

ResearcherID: Stephen Selkirk, MD, PhD: F-3655-2011 